\title{
Yerel Eğrilikli Dört Duvarlı Karbon Nanotüplerde Lineer Durumda Gerilme Dağılımı
}

\author{
Fatma Çoban KAYIKÇ I ${ }^{1 *}$ \\ 1* Yildiz Teknik Üniversitesi, Kimya Metalurji Fakültesi, İstanbul, Türkiye, (ORCID: 0000-0003-4417-7740), fatmacbn@ yildiz.edu.tr
}

(İlk Geliş Tarihi Aralık 2020 ve Kabul Tarihi Ocak 2021)

(DOI: 10.31590/ejosat.848831)

ATIF/REFERENCE: Çoban Kayıkçı, F. (2021). Yerel Eğrilikli Dört Duvarlı Karbon Nanotüplerde Lineer Durumda Gerilme Dağılımı. Avrupa Bilim ve Teknoloji Dergisi, (22), 167-175.

\section{$\ddot{O} \mathbf{z}$}

Son derece yüksek mukavemete sahip olan nanotüpler, nanoteknolojik uygulamalarda kullanılan nanomalzemelerin en önemli yapısal elemanlarıdır ve nanokompozit malzemelerin üretiminde kullanılırlar. Kompozitlerin matris malzemelerine yerleştirilerek üstün mekanik özellikler kazanmalarını sağlarlar. Kompozit malzemeler güçlendirilirken, yapısal nedenlerden veya teknolojik işlemlerden dolayı oluşan ilkel eğrilik, kendi kendini dengeleyen gerilmelerin ortaya çıkmasına neden olur. Bu gerilmelerin büyüklükleri, malzemenin güvenlik sınırlarının aşılmasına neden olabilir. Bu nedenle malzemelerin yük altında mekanik davranışlarının teorik olarak incelenmesi mühendislik açısından önemlidir. Bu çalı̧̧ada, yerel eğrilikli dört duvarlı karbon nanotüp içeren kompozit malzeme, üç boyutlu elastisite teorisinin kesin geometrik lineer denklemleri kullanılarak parçalı homojen cisim modeli kapsamında incelenmiştir. Çözüm için sınır formu pertürbasyon yöntemi bu probleme adapte edilerek yaklaşık analitik bir metot geliştirilmiştir. Çalışma çözüm yönteminin sıfırıncı ve birinci yaklaşımı için çözümü kapsamaktadır ve yeterlidir. Araştırma, karbon nanotüpün (CNT) dış katmanının dış yüzeyi ile matris malzemesinin kesişim yüzeyindeki normal ve kayma gerilmelerinin analizini içerir. Ayrıca karbon nanotüp ile matris arasında ideal temas koşulları kullanılmıştır. Sonuçlar, geometrik lineer durum için araştırılmıştır. Karbon nanotüp katmanları arasında ortaya çıkan Van der Waals kuvvetleri dikkate alınmış ve gerilmeler üzerindeki etkisi sayısal sonuçlar ile açıklanmıştır. Diğer problem parametrelerinin de gerilme dağılımı üzerindeki etkisine ilişkin sayısal sonuçlar sunulmuş ve tartışılmıştır. Ayrıca nanotüp en dış yarıçapının artması ile gerilim değerlerinin monoton olmayan şekilde değiştiği gözlenmiştir. Bununla birlikte, elastisite sabitlerinin oranının artmasının gerilmeleri önemli ölçüde etkilediği tespit edilmiştir. Karbon nano tüpün orta çizgisinin denkleminde bulunan ve salınım frekansını gösteren parametreye göre değişim de araştırılmış ve gerilmeler üzerindeki etkisi sunulmuştur. Bunun dışında önemli bir bulgu olarak karbon nanotüplerde de duvar sayısının artmasının gerilme değerlerini düşürdüğü tespit edilmiştir.

\section{Stress Distribution in Linear State in Four-Walled Carbon Nanotubes with Local Curvature}

\begin{abstract}
Nanotubes, which have extremely high strength, are the most important structural elements of nanomaterials used in nanotechnological applications and are used in the production of nanocomposite materials. They enable composites to gain superior mechanical properties by being placed in matrix materials. When composite materials are being strengthened, the primitive curvature caused by structural reasons or technological processes causes self-balancing stresses to appear. The magnitude of these stresses can cause the safety limits of the material to be exceeded. Therefore, it is important for engineering to examine the mechanical behavior of materials under load in terms of theoretically. In this study, the composite material containing four-walled carbon nanotube with local curvature was investigated within the scope of the piecewise homogeneous body model using the geometrical linear exact equations of the three-dimensional elasticity theory. An approximate analytical method has been developed for the solution by using the
\end{abstract}

*Sorumlu Yazar: fatmacbn@yildiz.edu.tr 
boundary form perturbation method. The study covers the solution for the zero and first approximation of the solution method and it is sufficient. The research involves the analysis of normal and shear stresses at the intersection surface of the matrix material with the outer surface of the carbon nanotube (CNT) outer layer. In addition, ideal contact conditions between the carbon nanotube and the matrix were used. The results were investigated for the geometric linear case. Van der Waals forces occurring between carbon nanotube layers were taken into account and their effect on stresses was explained with numerical results. Numerical results regarding the effect of other problem parameters on the stress distribution are presented and discussed. In addition, it has been observed that the stress values change non-monotonously with the increase of nanotube outermost radius. Moreover, it has been found that increasing the ratio of elasticity constants significantly affects the stresses. The change of the parameter showing the oscillation frequency in the equation of the centerline of the carbon nanotube is also investigated and its effect on stresses is presented. Apart from this, as an important finding, it has been determined that increasing the number of walls in carbon nanotubes decreases the stress values.

Keywords: Carbon nanotubes, Stress analysis, Local curvature.

\section{Giriş}

1991 yilında karbon nanotüplerin (CNT) keşfi (Iijima, 1991), polimer kompozitlerin matris malzemelerine eklenerek mekanik özelliklerinin artırılmasını sağlamıştır (Mantazeri ve Naghdabadi, 2009). Teorik ve deneysel olarak karbon nanotüplerin son derece yüksek sertliğe ve mukavemete sahip olduğu gösterilmiştir (Breuer ve Sundararaj, 2004). Ayrıca nanotüplerin bu özellikleri, havacılık sanayi, malzeme ve imalat sanayi, spor malzemeleri, savunma sanayi, biyoteknoloji, kompozit aynalar, otomotiv parçaları gibi çeşitli uygulamalarda avantaj sağlamaktadır.

Nano seviyedeki performans ve kontrol deneyleri çok pahalı ve zor bir iş olduğundan, bilim adamları ve mühendisler dikkatlerini teorik modellerin geliştirilmesine yöneltmektedir (Karličić ve diğerleri, 2017). CNT'lerin mekanik özelliklerinin teorik çalışmaları için kullanılan moleküler dinamik metodu, moleküler mekanik modeli, sonlu eleman modeli ve klasik süreklilik modeli gibi çeşitli metotlar vardır (Shi ve diğerleri, 2012: 1). Moleküler dinamik simülasyon, ayrık yapıları nedeniyle nanomalzemelerin analizi için diğer yöntemlere göre daha uygun olsa da, nano ölçekte deney yapmak zordur. Ayrıca atomistik modelleme, büyük boyutlu atomik sistemler için hesaplama açısından pahalıdır. Bu nedenle, sürekli modelleme, nanoyapıların mekanik özelliklerinin analizinde önemli bir role sahiptir (Arefi ve Nahvi, 2017). Nano yapıların incelenmesinde süreklilik mekaniğinin uygulanabilirliği (Guz, 2012 ve Duan ve diğerleri, 2009) 'da tartışılmıştır.

Birçok deneysel gözlemden elde edilen sonuca göre, CNT'ler (çoğu nano yapı) çoğunlukla eksenel yönleri boyunca belirli bir dalgalanma derecesiyle tanımlanır. Bu geometrik kusur, karbon nanotüplerin mekanik davranışı üzerinde önemli bir etkiye sahiptir. Başlangıç kusurları olan CNT'lerin düz olanlardan farklı mekanik davranışları vardır (Arefi and Nahvi, 2017). Kusurlar tübülün kıvrılmasına neden olur ve CNT'ler şimdiye kadar gözlemlenen ve sentezlenen düz, dalgalı, sarmal ve dallı gibi çeşitli tübül formlarına sahiptir (Zhang ve Li, 2009). Eğrilikli nanotüpler, mühendislik alanlarındaki birçok uygulamada yaygın olarak kullanılmaktadır (Mantazeri ve Naghdabadi, 2009). Çok duvarlı karbon nanotüpler (MWCNT) için de benzer çalışmalar mevcuttur ve genellikle MWCNT'ler kabuk veya kolon olarak modellenmiştir. Örneğin (Ru, 2001) 'de çift duvarlı CNT yerleştirilmiş elastik cismin eksenel basınç altında burkulması çalışılmıştır. (Yan ve diğerleri, 2010)' de, üç duvarlı karbon nanotüplerin (TWCNT) davranışı başlangıç eksenel gerilme altında incelenmiştir. Ayrıca, TWCNT' lerin üç elastik kabuktan oluştuğu ve van der Waals kuvvetleri ile bağlandıkları varsayılmıştır.
Kompozit malzemelerdeki bu bahsedilen ilkel eğrilik durumu, periyodik eğrilikli ve yerel eğrilikli olarak düşünülebilir. Periyodik eğrilikler tasarım sırasında oluşurken yerel eğrilikler teknolojik işlemler neticesinde ortaya çıkar. Kompozit malzemelerin üretimden sonra uygulamada başarılı olabilmesi, bahsedilen bu eğriliğin hesaplamalara dahil edilerek, malzemedeki gerilme-şekil değiştirme bağıntılarının belirlenmesine de bağlıdır. (Akbarov ve Kosker, 2001, 2003a, 2003b, 2003c, 2004), ve (Akbarov ve diğerleri., 2006, 2011) kaynaklarından görülebileceği üzere, bahsedilen eğrilikler kendini dengeleyen gerilmelerin ortaya çıkmasına sebep olmaktadır. Bu gerilmeler, eğriliğe ve diğer ilgili mekanik parametrelere bağlı olarak oldukça büyük değerlere ulaşıp kompozitin mukavemet sınırını aşabilmektedir. Bundan ve diğer sebeplerden ötürü, uygun fiziksel problemlerin matematiksel modellerinin yapılıp teorik olarak araştırılması, teorik açıdan da kompozitlerin uygulaması bakımından da oldukça önemlidir. (Coban, 2009)'de sonsuz ortam içerisinde düşük yoğunluklu sonsuz uzunluklu ve tek katlı karbon nanotüp olması durumu ele alınmış, çeşitli parametrelere bağlı olarak gerilme dağılımı incelenmiştir. Çalışma, üç boyutlu elastisite teorisi ile parçalı homojen cisim modeli kapsamında yapılmıştır. (Coban, 2016)'de iki katlı ve üç katlı CNT içeren elastik cismin gerilme ve stabilite analizi yapılmıştır.

Bu çalışmada ise literatürde yer almayan dört duvarlı karbon nanotüplerin üç boyutlu elastisite teorisine ait kesin geometrik nonlineer denklemler kullanılıp, parçalı-homojen cisim modeli çerçevesinde gerilme analizi ele alınmıştır.

\section{Materyal ve Metot}

\subsection{Matematiksel Yöntemin Geliştirilmesi}

Bu çalışmada, sonsuz uzunluklu, düşük yoğunluklu, sonsuz elastik cisme gömülü yerel eğrilikli çok duvarlı karbon nanotüp (MWCNT) göz önüne alınmıştır. Şekil 1'de olduğu gibi, MWCNT dört katmanlı olarak düşünülmüş ve dört içi boş yerel eğriliğe sahip silindirin eş merkezli olarak iç içe yerleştirilmesiyle modellenmiştir. Ayrıca silindirlerin arasında boşluk olduğu varsayılmıştır. Silindirlerin bitişik yüzeylerinde van der Waals kuvvetleri dikkate alınmıştır (Akbarov, 2013). Bahsedilen modelde, karbon nanotüpün uzunlamasına yönünde $(\mathrm{Oz})$ homojen olarak dağılmış, p yoğunluğuna sahip normal kuvvetlerin etkisinde olduğu varsayılmaktadır. Buna ek olarak, karbon nanotüpün yüzeyine dik olan bölümlerin yarıçaplarının nanotüp boyunca sabit kaldığı varsayılmaktadır. Çalışmadaki karbon nanotüp, sürekli bir malzeme olarak kabul edilmektedir (Kalamkarov, 2006). 


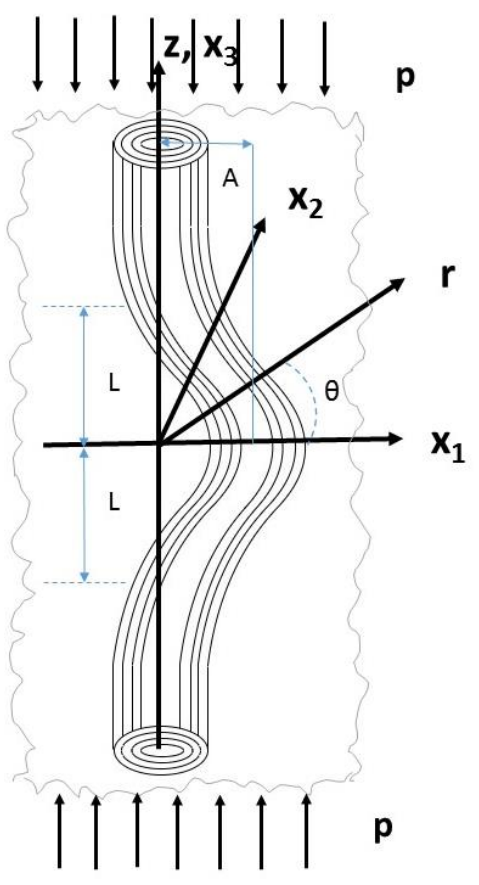

(a)

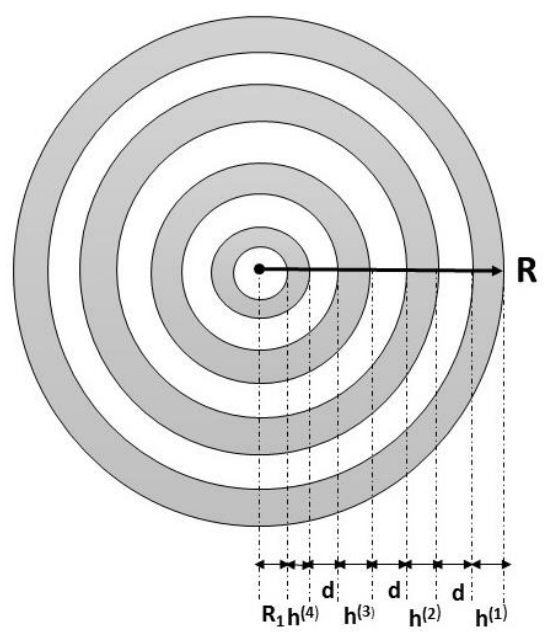

(b)

Şekil 1. Dört duvarlı CNT içeren sonsuz elastik cismin geometrisi (a) ve enine kesiti (b)

Koordinat sistemi olarak $\mathrm{Or} \theta \mathrm{z}$ silindirik ve $\mathrm{Ox}_{1} \mathrm{x}_{2} \mathrm{x}_{3}$ kartezyen koordinat sistemleri seçilmiş ve bu koordinatlar Lagrange koordinatları olarak kabul edilmiştir. Şekil 1'de gösterildiği gibi koordinatların başlangıcı iç içe geçmiş nanotüplerin (MWCNT) merkez çizgisi üzerinde olacak şekilde yerleştirilmiştir. Nanotüp yönünde $\left(\mathrm{Ox}_{3}(\mathrm{Oz})\right.$ yönünde) düzgün dağılmış normal kuvvetlerin, yukarıda modellenen sonsuz elastik cismi etkilediği düşünülmektedir. Ayrıca, $\mathrm{R}_{1}=\mathrm{R}-\mathrm{h}^{(1)}-3 \mathrm{~d}-\mathrm{h}^{(2)}-\mathrm{h}^{(3)}-\mathrm{h}^{(4)}$,

$\mathrm{R}_{2}=\mathrm{R}-\mathrm{h}^{(1)}-3 \mathrm{~d}-\mathrm{h}^{(2)}-\mathrm{h}^{(3)} \quad \mathrm{R}_{3}=\mathrm{R}-\mathrm{h}^{(1)}-2 \mathrm{~d}-\mathrm{h}^{(2)}-\mathrm{h}^{(3)}$, $\mathrm{R}_{4}=\mathrm{R}-\mathrm{h}^{(1)}-2 \mathrm{~d}-\mathrm{h}^{(2)}$, $\mathbf{R}_{5}=\mathbf{R}-\mathbf{h}^{(1)}-\mathrm{d}-\mathbf{h}^{(2)}$, $\mathrm{R}_{6}=\mathrm{R}-\mathrm{h}^{(1)}-\mathrm{d} \mathrm{R}_{7}=\mathrm{R}-\mathrm{h}^{(1)}, \quad \mathrm{R}_{8}=\mathrm{R} \quad$ yarıçaplarının nanotüpün merkez hattına dik dairesel kesitleri olduğu ve tüp boyunca sabit kaldığı varsayılmaktadır. Dört duvarlı olarak tasarlanan karbon nanotüp (MWCNT) ile matris malzemelerinin birbirinden farklı ve lineer elastik olduğu varsayılıp, incelemeler sürekli ortamlar mekaniğinin kesin geometrik lineer üç boyutlu denklemleri uygulanarak yapılmıştır. Şekil 1' deki cismin geometrisini göz önüne alarak nanotüpün ortak merkez (orta) çizgisinin denklemi aşağıdaki gibi dikkate alınmıştır.

$$
\mathrm{x}_{1}=\mathrm{F}\left(\mathrm{x}_{3}\right)=\varepsilon \delta\left(\mathrm{x}_{3}\right), \mathrm{x}_{2}=0
$$

Belirtmek gerekir ki (1)' deki $\varepsilon, 0 \leq \varepsilon<1$ olmak üzere, karbon nanotüpün eğilme genliğini ifade eden küçük bir parametredir. $\delta\left(\mathrm{x}_{3}\right)$ fonksiyonu ise karbon nanotüpün yüklemeden önceki eğilmesinin formunu göstermektedir. (1) denkleminden görüldüğü gibi, başlangıç yerel eğriliğe sahip dört duvarlı karbon nanotüpün orta çizgisi $\mathrm{x}_{2}=0$ düzlemi üzerindedir. Yüklemeden sonra da karbon nanotüpün orta çizgisinin bu düzlem üzerinde kaldığı varsayılacaktır. (1) ile verilen nanotüpün orta çizgisinin denklemi ve nanotüp kesitinin sağladığ 1 koşuldan yararlanarak, (Akbarov ve Güz, 2000) kaynağında gösterildiği gibi nanotüp ve matris ara yüzeyi olan $\mathrm{S}_{8}$ 'in (nanotüpün en diş yüzeyi) denklemi aşağıdaki gibi elde edilebilmektedir:

$$
\begin{gathered}
\mathrm{r}\left(\theta, \mathrm{t}_{3}\right)=\frac{\varepsilon \delta\left(\mathrm{t}_{3}\right)\left(1+\varepsilon^{2}\left(\delta^{\prime}\left(\mathrm{t}_{3}\right)\right)^{2}\right) \cos \theta}{1+\left(\delta^{\prime}\left(\mathrm{t}_{3}\right)\right)^{2} \varepsilon^{2} \cos ^{2} \theta}+ \\
\left\{\frac{\varepsilon^{2}\left(\delta\left(\mathrm{t}_{3}\right)\right)^{2}\left(1+\varepsilon^{2}\left(\delta^{\prime}\left(\mathrm{t}_{3}\right)\right)^{2}\right)^{2} \cos \theta}{\left(1+\left(\delta^{\prime}\left(\mathrm{t}_{3}\right)\right)^{2} \varepsilon^{2} \cos ^{2} \theta\right)^{2}}+\mathrm{R}^{2}-\left(\delta\left(\mathrm{t}_{3}\right)\right)^{2} \varepsilon^{2}\left(1+\varepsilon^{2}\left(\delta^{\prime}\left(\mathrm{t}_{3}\right)\right)^{2}\right)\right\}^{1 / 2} \\
\mathrm{x}_{3}\left(\theta, \mathrm{t}_{3}\right)=\mathrm{t}_{3}-\varepsilon \delta^{\prime}\left(\mathrm{t}_{3}\right)\left(\mathrm{r}\left(\theta, \mathrm{t}_{3}\right)-\varepsilon \delta\left(\mathrm{t}_{3}\right)\right), \delta^{\prime}\left(\mathrm{t}_{3}\right)=\frac{\mathrm{d} \delta\left(\mathrm{t}_{3}\right)}{\mathrm{dt}_{3}} .
\end{gathered}
$$

Burada $t_{3}$ bir parametredir ve $t_{3} \in(-\infty,+\infty)$, dir. (2) denklemleri aracılı̆̆ $\breve{1}_{1}$ ile $S_{8}$ yüzeyinin birim diş normalinin bileşenleri için aşağıdaki denklemleri elde edilir (Akbarov ve Güz, 2000):

$$
\begin{aligned}
& \mathrm{n}_{\mathrm{r}}=\mathrm{r}\left(\theta, \mathrm{t}_{3}\right) \frac{\partial \mathrm{z}\left(\theta, \mathrm{t}_{3}\right)}{\partial \mathrm{t}_{3}}[\mathrm{~A}(\theta, \mathrm{z})]^{-1} \\
& \mathrm{n}_{\theta}=\left[\frac{\partial \mathrm{z}\left(\theta, \mathrm{t}_{3}\right)}{\partial \theta} \frac{\partial \mathrm{r}\left(\theta, \mathrm{t}_{3}\right)}{\partial \mathrm{t}_{3}}-\frac{\partial \mathrm{r}\left(\theta, \mathrm{t}_{3}\right)}{\partial \theta} \frac{\partial \mathrm{z}\left(\theta, \mathrm{t}_{3}\right)}{\partial \mathrm{t}_{3}}\right][\mathrm{A}(\theta, \mathrm{z})]^{-1} \\
& \mathrm{n}_{\mathrm{z}}=\mathrm{r}\left(\theta, \mathrm{t}_{3}\right) \frac{\partial \mathrm{z}\left(\theta, \mathrm{t}_{3}\right)}{\partial \mathrm{t}_{3}}\left[\mathrm{~A}\left(\theta, \mathrm{t}_{3}\right)\right]^{-1}
\end{aligned}
$$

Burada,

$$
\mathrm{A}\left(\theta, \mathrm{t}_{3}\right)=\left[\begin{array}{l}
\left(\mathrm{r}\left(\theta, \mathrm{t}_{3}\right) \frac{\partial \mathrm{z}\left(\theta, \mathrm{t}_{3}\right)}{\partial \mathrm{t}_{3}}\right)^{2}+\left(\mathrm{r}\left(\theta, \mathrm{t}_{3}\right) \frac{\partial \mathrm{z}\left(\theta, \mathrm{t}_{3}\right)}{\partial \mathrm{t}_{3}}\right)^{2}+ \\
\left(\frac{\partial \mathrm{z}\left(\theta, \mathrm{t}_{3}\right)}{\partial \theta} \frac{\partial \mathrm{r}\left(\theta, \mathrm{t}_{3}\right)}{\partial \mathrm{t}_{3}}-\frac{\partial \mathrm{z}\left(\theta, \mathrm{t}_{3}\right)}{\partial \mathrm{t}_{3}} \frac{\partial \mathrm{r}\left(\theta, \mathrm{t}_{3}\right)}{\partial \theta}\right)^{2}
\end{array}\right]^{1 / 2}
$$

Şeklindedir. Bu aşamadan itibaren; matris malzemesi ile ilgili büyüklükler (1), dört duvarlı karbon nanotüpün en dış katmanı ile ilgili büyüklükler (2), dıştan ikinci katmanı ile ilgili büyüklükler (3), bir içteki katman ile ilgili büyüklükler (4) ve en iç katmanı ile ilgili büyüklükler (5) üst indisleriyle gösterilecektir. Ele alınan çok duvarlı karbon nanotüpün tüm katmanlarında ve matris malzemesinin her birinde sağlanmak koşuluyla; denge denklemleri, şekil değiştirme-yer değiştirme ilişkileri ve bünye denklemlerinin sağlandığı varsayılacaktır:

$$
\nabla_{\mathrm{i}}\left[\sigma^{(\mathrm{k}) \mathrm{in}}\left(\mathrm{g}_{\mathrm{n}}^{\mathrm{j}}+\nabla_{\mathrm{n}} \mathrm{u}^{(\mathrm{k}) \mathrm{j}}\right)\right]=0
$$




$$
\begin{aligned}
& 2 \varepsilon_{\mathrm{jm}}^{(\mathrm{k})}=\nabla_{\mathrm{j}} \mathrm{u}_{\mathrm{m}}^{(\mathrm{k})}+\nabla_{\mathrm{m}} \mathrm{u}_{\mathrm{j}}^{(\mathrm{k})}+\nabla_{\mathrm{j}} \mathrm{u}^{(\mathrm{k}) \mathrm{n}} \nabla_{\mathrm{m}} \mathrm{u}^{(\mathrm{k}) \mathrm{n}} \\
& \sigma_{(\mathrm{ip})}^{(\mathrm{k})}=\left(\lambda^{(\mathrm{k})} \mathrm{e}^{(\mathrm{k})}\right) \delta_{\mathrm{i}}^{\mathrm{p}}+2\left(\mu^{(\mathrm{k})} \varepsilon_{(\mathrm{ip})}^{(\mathrm{k})}\right) \\
& \mathrm{e}^{(\mathrm{k})}=\varepsilon_{11}^{(\mathrm{k})}+\varepsilon_{22}^{(\mathrm{k})}+\varepsilon_{33}^{(\mathrm{k})}
\end{aligned}
$$

Burada (5)' de verilen $\sigma_{(\text {in) }}^{(\mathrm{k})}$ 'lar ve $\varepsilon_{(\mathrm{in})}^{(\mathrm{k})}$ 'lar sirasiyla gerilme ve şekil değiștirme tansörlerinin fiziksel bileșenlerini ifade etmektedir. Aynı zamanda karbon nanotüp ve matris ara yüzeyi olan $\mathrm{S}_{8}$ yüzeyinde ideal temas koşullarının sağlandığ varsayılacaktır. $S_{8}$ yüzeyinin dış normal vektörü bileşenleri $\mathbf{n}_{j}$ olmak üzere bu koşullar aşağıdaki gibi verilmektedir:

$$
\begin{aligned}
& \left.\sigma^{(1) i p}\left(g_{p}^{j}+\nabla_{p} u^{(1) j}\right)\right|_{S_{8}} n_{j}=\left.\sigma^{(2) i p}\left(g_{p}^{j}+\nabla_{p} u^{(2) j}\right)\right|_{S_{8}} n_{j}, \\
& \left.u^{(1) j}\right|_{S_{8}}=\left.u^{(2) j}\right|_{S_{8}}
\end{aligned}
$$

En diştaki tüpün iç yüzeyi $\left(\mathrm{S}_{7}\right)$ ile dıştan ikinci tüpün diş yüzeyi $\left(\mathrm{S}_{6}\right)$ arasındaki sınır koşulları $\mathrm{n}_{\mathrm{j}}^{(\mathrm{k})}(\mathrm{k}=2,3)$ yüzeylerinin dış normalleri olmak üzere aşağıdaki gibi yazılabilir:

$$
\begin{aligned}
& \left.\left(\mathrm{R}_{7}\right) \sigma^{(2) p p}\left(\mathrm{~g}_{\mathrm{p}}^{\mathrm{j}}+\nabla_{\mathrm{p}} \mathrm{u}^{(2) j}\right)\right|_{\mathrm{S}_{7}} \mathrm{n}_{\mathrm{j}}^{(\mathrm{k})}=\mathrm{c}\left(\left.\mathrm{u}_{\mathrm{p}}^{(2)}\right|_{\mathrm{s}_{7}}-\left.\mathrm{u}_{\mathrm{p}}^{(3)}\right|_{\mathrm{S}_{6}}\right) \\
& \left.\sigma^{(2) i p}\left(\mathrm{~g}_{\mathrm{p}}^{\mathrm{j}}+\nabla_{\mathrm{p}} \mathrm{u}^{(2) \mathrm{j}}\right)\right|_{\mathrm{S}_{7}} \mathrm{n}_{\mathrm{j}}^{(\mathrm{k})}=0, \mathrm{i}=\tau, \mathrm{e} \\
& \left.\left(\mathrm{R}_{7}\right) \sigma^{(2) p p}\left(\mathrm{~g}_{\mathrm{p}}^{\mathrm{j}}+\nabla_{\mathrm{p}} \mathrm{u}^{(2) j}\right)\right|_{\mathrm{S}_{7}} \mathrm{n}_{\mathrm{j}}^{(\mathrm{k})}=\left.\left(\mathrm{R}_{6}\right) \sigma^{(3) p p}\left(\mathrm{~g}_{\mathrm{p}}^{\mathrm{j}}+\nabla_{\mathrm{p}} \mathrm{u}^{(3) \mathrm{j}}\right)\right|_{\mathrm{S}_{6}} \mathrm{n}_{\mathrm{j}}^{(\mathrm{k})} \\
& \left.\sigma^{(3) \mathrm{p} p}\left(\mathrm{~g}_{\mathrm{p}}^{\mathrm{j}}+\nabla_{\mathrm{p}} \mathrm{u}^{(3) j}\right)\right|_{\mathrm{S}_{4}} \mathrm{n}_{\mathrm{j}}^{(\mathrm{k})}=0, \mathrm{i}=\tau, \mathrm{e}
\end{aligned}
$$

D1ştan ikinci tüpün iç yüzeyi $\left(\mathrm{S}_{5}\right)$ ile dıştan üçüncü tüpün dış yüzeyi $\left(\mathrm{S}_{4}\right)$ arasındaki sınır koşulları $\mathrm{n}_{\mathrm{j}}^{(\mathrm{k})} \quad(\mathrm{k}=3,4)$ yüzeylerinin dış normalleri olmak üzere aşağıdaki gibi yazılabilir:

$$
\begin{aligned}
& \left.\left(\mathbf{R}_{5}\right) \sigma^{(3) p p}\left(g_{p}^{j}+\nabla_{p} u^{(3) j}\right)\right|_{S_{5}} n_{j}^{(k)}=c\left(\left.u_{p}^{(3)}\right|_{S_{5}}-\left.u_{p}^{(4)}\right|_{S_{4}}\right) \\
& \left.\sigma^{(3) i p}\left(g_{p}^{j}+\nabla_{p} u^{(3) j}\right)\right|_{S_{5}} n_{j}^{(k)}=0, i=\tau, e \\
& \left.\left(R_{5}\right) \sigma^{(3) p p}\left(g_{p}^{j}+\nabla_{p} u^{(3) j}\right)\right|_{S_{5}} n_{j}^{(k)}= \\
& \left.\left(R_{4}\right) \sigma^{(4) p p}\left(g_{p}^{j}+\nabla_{p} u^{(4) j}\right)\right|_{S_{4}} n_{j}^{(k)} \\
& \left.\sigma^{(4) i p}\left(g_{p}^{j}+\nabla_{p} u^{(3) j}\right)\right|_{S_{4}} n_{j}^{(k)}=0, i=\tau, e
\end{aligned}
$$

D1ştan üçüncü tüpün iç yüzeyi $\left(\mathrm{S}_{3}\right)$ ile en içteki tüpün dış yüzeyi $\left(\mathrm{S}_{2}\right)$ arasındaki sınır koşulları $\mathrm{n}_{\mathrm{j}}^{(\mathrm{k})}(\mathrm{k}=4,5)$ yüzeylerinin dış normalleri olmak üzere aşağıdaki gibi yazılabilir:

$$
\begin{aligned}
& \left.\left(\mathbf{R}_{3}\right) \sigma^{(4) p p}\left(g_{p}^{j}+\nabla_{p} u^{(4) j}\right)\right|_{S_{3}} n_{j}{ }^{(k)}=c\left(\left.u_{p}^{(4)}\right|_{S_{3}}-\left.u_{p}^{(5)}\right|_{S_{2}}\right) \\
& \left.\sigma^{(4) \text { ip }}\left(g_{p}^{j}+\nabla_{p} u^{(4) j}\right)\right|_{S_{3}} n_{j}{ }^{(k)}=0, \quad i=\tau, e
\end{aligned}
$$

$$
\begin{aligned}
& \left.\left(R_{3}\right) \sigma^{(4) p p}\left(g_{p}^{j}+\nabla_{p} u^{(4) j}\right)\right|_{S_{3}} n_{j}^{(k)}= \\
& \left.\left(R_{2}\right) \sigma^{(5) p p}\left(g_{p}^{j}+\nabla_{p} u^{(5) j}\right)\right|_{S_{2}} n_{j}^{(k)} \\
& \left.\sigma^{(5) i p}\left(g_{p}^{j}+\nabla_{p} u^{(5) j}\right)\right|_{S_{2}} n_{j}^{(k)}=0, i=\tau, e
\end{aligned}
$$

En içteki tüpün iç yüzeyindeki $\left(\mathrm{S}_{1}\right)$ sınır koşulları ise aşağıdaki gibi yazılabilir:

$$
\left.\sigma^{(5) i p}\left(g_{p}^{j}+\nabla_{p} u^{(5) j}\right)\right|_{S_{1}} n_{j}^{(k)}=0
$$

Ayrica,

$$
\sigma_{\mathrm{zz}}^{(1)} \underset{\mathrm{z} \rightarrow \infty}{\rightarrow} \mathrm{p}, \sigma_{\mathrm{ij}}^{(1)} \underset{\mathrm{r} \rightarrow \infty}{\rightarrow} 0,(\mathrm{ij})=\mathrm{rr}, \mathrm{r} \theta, \mathrm{rz}, \theta \theta, \theta \mathrm{z}, \mathrm{zz}
$$

koşullarının sağlandığı da varsayılacaktır.

$\mathrm{Bu}$ şekilde ele alınan fiziksel problemin matematik formülasyonu genel hatlarıyla verilmiş olmaktadır. Böylece, sonsuz elastik bir ortamdaki yerel eğrilikli sonsuz uzun dört duvarlı karbon nanotüp içeren kompozit malzemenin nanotüpün boyuna yönünde etki eden dış kuvvetler altında incelenmesi, (5) denklemlerinin (6)-(11) sınır koşulları çerçevesinde araştırılmasına getirilmektedir.

\section{2. Çözüm Yönteminin Geliştirilmesi}

Önceki bölümde matematiksel formülasyonu verilen, nonlineer kısmi diferansiyel denklem takımı haline gelen sınırdeğer probleminin çözümü için, (Akbarov ve Guz, 2000) ve (Akbarov ve Guz, 1985)'de detayları verilmiş olan sınır formu pertürbasyon yöntemi kullanılacaktır. $\mathrm{Bu}$ yöntemde aranan büyüklükler (1)' de verilen karbon nanotüpün orta çizgisinin denkleminde yer alan ve onun eğilme derecesini gösteren oldukça küçük $\varepsilon$ parametresinin serisi halinde (12)'de olduğu gibi yazılabilmektedir.

$\sigma_{\text {rr }}^{(\mathrm{k})}=\sum_{\mathrm{q}=0}^{\infty} \varepsilon^{\mathrm{q}} \sigma_{\mathrm{rr}}^{(\mathrm{k}), \mathrm{q}} \varepsilon_{\mathrm{rr}}^{(\mathrm{k})}=\sum_{\mathrm{q}=0}^{\infty} \varepsilon^{\mathrm{q}} \varepsilon_{\mathrm{rr}}^{(\mathrm{k}), \mathrm{q}} \mathrm{u}_{\mathrm{r}}^{(\mathrm{k})}=\sum_{\mathrm{q}=0}^{\infty} \varepsilon^{\mathrm{q}} \mathrm{u}_{\mathrm{r}}^{(\mathrm{k}), \mathrm{q}}$

(12)'deki ifadeler (5) denklemlerinde yerlerine yazılır, $\varepsilon^{\mathrm{k}}$ 'nın katsayıları $\varepsilon$ 'nun aynı derecelerine göre gruplandırılır ve $\left(\mathrm{R}, \theta, \mathrm{t}_{3}\right)$ civarında seriye açılırsa her bir yaklaşım için $\mathrm{S}_{1}$, $\mathrm{S}_{2}, \ldots, \mathrm{S}_{8}$ yüzeylerinde sağlanan temas koşulları elde edilir. Birinci ve daha sonraki yaklaşımlar için elde edilen denklemler önceki yaklaşımların büyüklüklerini de içermektedirler. $\mathrm{Bu}$ çalışmada sıfırıncı ve birinci yaklaşım için çözüm aranmıştır. Yapılan çalışmalarda ikinci ve daha sonraki yaklaşımların sayısal sonuçlar üzerinde önemli bir etkisi olmadığı görülmüştür (Akbarov ve Güz, 2000).

Sıfırıncı ve birinci yaklaşımın çözümünü ele alalım. Sadelik açısından $v^{(1)}$ matris malzemesinin, $v^{(2)}, v^{(3)}, v^{(4)}, v^{(5)}$ sırasıyla dıştan içeri doğru karbon nanotüpün katmanlarının Poisson oranları olmak üzere $v^{(1)}=v^{(2)}=v^{(3)}=v^{(4)}=v^{(5)}$ olduğunu varsayılacaktır. Poisson oranlarının eşit alınması, sayısal sonuçlara önemli bir etki yapmamaktadır (Akbarov ve Güz, 2000). Bu nedenle, sadece işlemleri kolaylaştırmak için Poisson oranları eşit alınmıştır. Sıfırıncı yaklaşım için (5) denklemlerinin çözümü aşağıdaki gibi elde edilir: 


$$
\begin{aligned}
& \varepsilon_{\mathrm{zz}}^{(1), 0}=\varepsilon_{\mathrm{zz}}^{(2), 0}=\varepsilon_{\mathrm{zz}}^{(3), 0}=\varepsilon_{\mathrm{zz}}^{(4), 0}=\varepsilon_{\mathrm{zz}}^{(5), 0}=\frac{\mathrm{p}}{\mathrm{E}^{(1)}}, \sigma_{\mathrm{zz}}^{(1), 0}=\mathrm{p}, \\
& u_{\mathrm{z}}^{(1), 0}=u_{\mathrm{z}}^{(2), 0}=u_{\mathrm{z}}^{(3), 0}=u_{\mathrm{z}}^{(4), 0}=u_{\mathrm{z}}^{(5), 0}=\frac{\mathrm{p}}{\mathrm{E}^{(1)}} \mathrm{z}, \\
& u_{\mathrm{r}}^{(5), 0}=-v^{(5)} \varepsilon_{\mathrm{zz}}^{(5), 0} \mathbf{r}, u_{\mathrm{r}}^{(4), 0}=-v^{(4)} \varepsilon_{\mathrm{zz}}^{(4), 0} \mathbf{r}, \\
& u_{\mathrm{r}}^{(3), 0}=-v^{(3)} \varepsilon_{\mathrm{zz}}^{(3), 0} \mathrm{r}, u_{\mathrm{r}}^{(2), 0}=-v^{(2)} \varepsilon_{\mathrm{zz}}^{(2), 0} \mathrm{r}, \\
& u_{\mathrm{r}}^{(1), 0}=-v^{(1)} \varepsilon_{\mathrm{zz}}^{(1), 0} \mathrm{r} \\
& u_{\theta}^{(1), 0}=u_{\theta}^{(2), 0}=u_{\theta}^{(3), 0}=u_{\theta}^{(4), 0}=u_{\theta}^{(5), 0}=0, \\
& \sigma_{\mathrm{rr}}^{(1), 0}=\sigma_{\mathrm{rr}}^{(2), 0}=\sigma_{\mathrm{rr}}^{(3), 0}=\sigma_{\mathrm{rr}}^{(4), 0}=\sigma_{\mathrm{rr}}^{(5), 0}=0, \\
& \sigma_{\theta \theta}^{(1), 0}=\sigma_{\theta \theta}^{(2), 0}=\sigma_{\theta \theta}^{(3), 0}=\sigma_{\theta \theta}^{(4), 0}=\sigma_{\theta \theta}^{(5), 0}=0, \\
& \sigma_{\mathrm{zz}}^{(2), 0}=\mathrm{p} \frac{\mathrm{E}^{(2)}}{\mathrm{E}^{(1)}}, \sigma_{\mathrm{zz}}^{(3), 0}=\mathrm{p} \frac{\mathrm{E}^{(3)}}{\mathrm{E}^{(1)}}, \\
& \sigma_{\mathrm{zz}}^{(4), 0}=\mathrm{p} \frac{\mathrm{E}^{(4)}}{\mathrm{E}^{(1)}}, \\
& \sigma_{\theta \mathrm{z}}^{(1), 0}=\sigma_{\theta \mathrm{z}}^{(2), 0}=\sigma_{\theta \mathrm{z}}^{(3), 0}=\sigma_{\theta \mathrm{z}}^{(4), 0}=\sigma_{\theta \mathrm{z}}^{(5), 0}=0, \\
& \sigma_{\mathrm{rz}}^{(1), 0}=\sigma_{\mathrm{rz}}^{(2), 0}=\sigma_{\mathrm{rz}}^{(3), 0}=\sigma_{\mathrm{rz}}^{(4), 0}=\sigma_{\mathrm{rz}}^{(5), 0}=0, \\
& \sigma_{\mathrm{r} \theta}^{(1), 0}=\sigma_{\mathrm{r} \theta}^{(2), 0}=\sigma_{\mathrm{r} \theta}^{(3), 0}=\sigma_{\mathrm{r} \theta}^{(4), 0}=\sigma_{\mathrm{r} \theta}^{(5), 0}=0
\end{aligned}
$$

Birinci yaklaşım için problemin çözümünü ele alalım. Yukarıda bahsedilen tüm kabuller çerçevesinde ve (13) ifadelerinin dikkate alınmasıyla (5)'deki denge denklemlerinin silindirik koordinatlardaki ifadesi aşağıdaki gibi elde edilir.

$$
\begin{aligned}
& \frac{\partial \sigma_{\mathrm{rr}}^{(1), 1}}{\partial \mathrm{r}}+\frac{1}{\mathrm{r}} \frac{\partial \sigma_{\mathrm{r} \theta}^{(1), 1}}{\partial \theta}+\frac{\partial \sigma_{\mathrm{rz}}^{(1), 1}}{\partial \mathrm{z}}+\frac{1}{\mathrm{r}}\left(\sigma_{\mathrm{rr}}^{(\mathrm{l}), 1}-\sigma_{\theta \theta}^{(1), 1}\right)=0 \\
& \frac{\partial \sigma_{\mathrm{r} \theta}^{(1), 1}}{\partial \mathrm{r}}+\frac{1}{\mathrm{r}} \frac{\partial \sigma_{\theta \theta}^{(1), 1}}{\partial \theta}+\frac{\partial \sigma_{\theta \mathrm{z}}^{(1), 1}}{\partial \mathrm{z}}+\frac{2}{\mathrm{r}} \sigma_{\mathrm{r} \theta}^{(1), 1}=0 \\
& \frac{\partial \sigma_{\mathrm{rz}}^{(1), 1}}{\partial \mathrm{r}}+\frac{1}{\mathrm{r}} \frac{\partial \sigma_{\theta \mathrm{z}}^{(1), 1}}{\partial \theta}+\frac{\partial \sigma_{\mathrm{zz}}^{(1), 1}}{\partial \mathrm{z}}+\frac{1}{\mathrm{r}} \sigma_{\mathrm{rz}}^{(1), 1}=0
\end{aligned}
$$

Elde edilen (14) denklemlerinin üç boyutlu lineerize edilmiş elastisite denklemleri oldukları görülür (Güz, 1999). Karbon nanotüpün orta çizgisinin denklemi,

$$
\begin{aligned}
& \mathrm{x}_{1}=\operatorname{Aexp}\left(-\left(\frac{\mathrm{x}_{3}}{\mathrm{~L}}\right)^{2}\right) \cos \left(\mathrm{m} \frac{\mathrm{x}_{3}}{\mathrm{~L}}\right)=\varepsilon \delta\left(\mathrm{x}_{3}\right) ; \\
& \varepsilon=\frac{\mathrm{A}}{\mathrm{L}}, \mathrm{L}>\mathrm{A}
\end{aligned}
$$

şeklinde tanımlanmıştır. Yukarıdaki ifadeler dikkate alınırsa, (6)(11) sınır koşulları ilk yaklaşım için aşağıdaki gibi olur:

$$
\begin{aligned}
& \left.\left(\sigma_{\mathrm{rr}}^{(1), 1}-\sigma_{\mathrm{rr}}^{(2), 1}\right)\right|_{\mathrm{r}=\mathrm{R}}=0,\left.\left(\sigma_{\mathrm{r} \theta}^{(1), 1}-\sigma_{\mathrm{r} \theta}^{(2), 1}\right)\right|_{\mathrm{r}=\mathrm{R}}=0, \\
& \left.\left(\sigma_{\mathrm{rz}}^{(1), 1}-\sigma_{\mathrm{rz}}^{(2), 1}\right)\right|_{\mathrm{r}=\mathrm{R}}=\left(\sigma_{\mathrm{zz}}^{(1), 0}-\sigma_{\mathrm{zz}}^{(2), 0}\right) \frac{\mathrm{d} \delta\left(\mathrm{t}_{3}\right)}{\mathrm{dt}_{3}} \cos \theta, \\
& \left.\left(\mathrm{u}_{\mathrm{r}}^{(1), 1}-\mathrm{u}_{\mathrm{r}}^{(2), 1}\right)\right|_{\mathrm{r}=\mathrm{R}}=0,\left.\left(\mathrm{u}_{\theta}^{(1), 1}-\mathrm{u}_{\theta}^{(2), 1}\right)\right|_{\mathrm{r}=\mathrm{R}}=0, \\
& \left.\left(\mathrm{u}_{\mathrm{z}}^{(1), 1}-\mathrm{u}_{\mathrm{z}}^{(2), 1}\right)\right|_{\mathrm{r}=\mathrm{R}}=0,
\end{aligned}
$$

$$
\begin{aligned}
& \left.\sigma_{\mathrm{r} \theta}^{(2), 1}\right|_{\mathrm{r}=\mathrm{R}-\mathrm{h}^{(1)}}=0 \text {, } \\
& \left.\left(\mathrm{R}-\mathrm{h}^{(1)}\right) \sigma_{\mathrm{rr}}{ }^{(2), 1}\right|_{\mathrm{r}=\mathrm{R}-\mathrm{h}^{(1)}}=\mathrm{c}\left(\left.\mathrm{u}_{\mathrm{r}}{ }^{(2)}\right|_{\mathrm{r}=\mathrm{R}-\mathrm{h}^{(1)}}-\left.\mathrm{u}_{\mathrm{r}}{ }^{(3)}\right|_{\mathrm{r}=\mathrm{R}-\mathrm{h}^{(1)}-\mathrm{d}}\right) \\
& \left.\sigma_{\mathrm{rz}}^{(2), 1}\right|_{\mathrm{r}=\mathrm{R}-\mathrm{h}^{(1)}}=\sigma_{\mathrm{zz}}^{(2), 0} \frac{\mathrm{d} \delta\left(\mathrm{t}_{3}\right)}{\mathrm{dt}_{3}} \cos \theta \text {, } \\
& \left.\left(\mathrm{R}-\mathrm{h}^{(1)}\right) \sigma_{\mathrm{rr}}{ }^{(2), 1}\right|_{\mathrm{r}=\mathrm{R}-\mathrm{h}^{(1)}}=\left.\left(\mathrm{R}-\mathrm{h}^{(1)}-\mathrm{d}\right) \sigma_{\mathrm{rr}}^{(3), 1}\right|_{\mathrm{r}=\mathrm{R}-\mathrm{h}^{(1)}-\mathrm{d}}, \\
& \left.\sigma_{\mathrm{rz}}^{(3), 1}\right|_{\mathrm{r}=\mathrm{R}-\mathrm{h}^{(1)}-\mathrm{d}}=\sigma_{\mathrm{zz}}^{(3), 0} \frac{\mathrm{d} \delta\left(\mathrm{t}_{3}\right)}{\mathrm{dt}_{3}} \cos \theta,\left.\quad \sigma_{\mathrm{r} \theta}^{(3), 1}\right|_{\mathrm{r}=\mathrm{R}-\mathrm{h}^{(1)}-\mathrm{d}}=0 \\
& \left.\left(\mathrm{R}-\mathrm{h}^{(1)}-\mathrm{h}^{(2)}-\mathrm{d}\right) \sigma_{\mathrm{rr}}^{(3), 1}\right|_{\mathrm{r}=\mathrm{R}-\mathrm{h}^{(1)}-\mathrm{h}^{(2)}-\mathrm{d}}=\mathrm{c}\left(\left.\mathrm{u}_{\mathrm{r}}^{(3)}\right|_{\mathrm{r}=\mathrm{R}-\mathrm{h}^{(1)}-\mathrm{h}^{(2)}-\mathrm{d}}\right. \\
& \left.-\left.\mathrm{u}_{\mathrm{r}}^{(4)}\right|_{\mathrm{r}=\mathrm{R}-\mathrm{h}^{(1)}-2 \mathrm{~d}-\mathrm{h}^{(2)}}\right) \\
& \left.\sigma_{\mathrm{r} \theta}^{(3), 1}\right|_{\mathrm{r}=\mathrm{R}-\mathrm{h}^{(1)}-\mathrm{h}^{(2)}-\mathrm{d}}=0 \text {, } \\
& \left.\sigma_{\mathrm{rz}}{ }^{(3), 1}\right|_{\mathrm{r}=\mathrm{R}-\mathrm{h}^{(1)}-\mathrm{h}^{(2)}-\mathrm{d}}=\sigma_{\mathrm{zz}}^{(3), 0} \frac{\mathrm{d} \delta\left(\mathrm{t}_{3}\right)}{\mathrm{dt}_{3}} \cos \theta \\
& \left.\left(\mathrm{R}-\mathrm{h}^{(1)}-\mathrm{h}^{(2)}-\mathrm{d}\right) \sigma_{\mathrm{rr}}{ }^{(3), 1}\right|_{\mathrm{r}=\mathrm{R}-\mathrm{h}^{(1)}-\mathrm{h}^{(2)}-\mathrm{d}}= \\
& \left.\left(\mathrm{R}-\mathrm{h}^{(1)}-\mathrm{h}^{(2)}-2 \mathrm{~d}\right) \sigma_{\mathrm{rr}}{ }^{(4), 1}\right|_{\mathrm{r}=\mathrm{R}-\mathrm{h}^{(1)}-\mathrm{h}^{(2)}-2 \mathrm{~d}} \\
& \left.\sigma_{\mathrm{r} \theta}{ }^{(4), 1}\right|_{\mathrm{r}=\mathrm{R}-\mathrm{h}^{(1)}-\mathrm{h}^{(2)}-2 \mathrm{~d}}=0 \text {, } \\
& \left.\sigma_{\mathrm{rz}}^{(4), 1}\right|_{\mathrm{r}=\mathrm{R}-\mathrm{h}^{(1)}-\mathrm{h}^{(2)}-2 \mathrm{~d}}=\sigma_{\mathrm{zz}}^{(4), 0} \frac{\mathrm{d} \delta\left(\mathrm{t}_{3}\right)}{\mathrm{dt}_{3}} \cos \theta \\
& \left.\left(\mathrm{R}-\mathrm{h}^{(1)}-\mathrm{h}^{(2)}-2 \mathrm{~d}-\mathrm{h}^{(3)}\right) \sigma_{\mathrm{rr}}^{(4), 1}\right|_{\mathrm{r}=\mathrm{R}-\mathrm{h}^{(1)}-\mathrm{h}^{(2)}-2 \mathrm{~d}-\mathrm{h}^{(3)}}= \\
& c\left(\left.u_{r}{ }^{(4)}\right|_{r=R-h^{(1)}-h^{(2)}-2 d-h^{(3)}}-\left.u_{r}^{(5)}\right|_{r=R-h^{(1)}-3 d-h^{(2)}-h^{(3)}}\right) \\
& \left.\sigma_{\mathrm{r} \theta}^{(4), 1}\right|_{\mathrm{r}=\mathrm{R}-\mathrm{h}^{(1)}-\mathrm{h}^{(2)}-2 \mathrm{~d}-\mathrm{h}^{(3)}}=0 \text {, } \\
& \left.\sigma_{\mathrm{rz}}^{(4), 1}\right|_{\mathrm{r}=\mathrm{R}-\mathrm{h}^{(1)}-\mathrm{h}^{(2)}-2 \mathrm{~d}-\mathrm{h}^{(3)}}=\sigma_{\mathrm{zz}}^{(4), 0} \frac{\mathrm{d} \delta\left(\mathrm{t}_{3}\right)}{\mathrm{dt}_{3}} \cos \theta, \\
& \left.\left(\mathrm{R}-\mathrm{h}^{(1)}-\mathrm{h}^{(2)}-2 \mathrm{~d}-\mathrm{h}^{(3)}\right) \sigma_{\mathrm{rr}}{ }^{(4), 1}\right|_{\mathrm{r}=\mathrm{R}-\mathrm{h}^{(1)}-\mathrm{h}^{(2)}-2 \mathrm{~d}-\mathrm{h}^{(3)}}= \\
& \left.\left(\mathrm{R}-\mathrm{h}^{(1)}-\mathrm{h}^{(2)}-3 \mathrm{~d}-\mathrm{h}^{(3)}\right) \sigma_{\mathrm{rr}}{ }^{(5), 1}\right|_{\mathrm{r}=\mathrm{R}-\mathrm{h}^{(1)}-\mathrm{h}^{(2)}-3 \mathrm{~d}-\mathrm{h}^{(3)}} \\
& \left.\sigma_{\mathrm{r} \theta}^{(5), 1}\right|_{\mathrm{r}=\mathrm{R}-\mathrm{h}^{(1)}-\mathrm{h}^{(2)}-3 \mathrm{~d}-\mathrm{h}^{(3)}}=0 \text {, } \\
& \left.\sigma_{\mathrm{rz}}^{(5), 1}\right|_{\mathrm{r}=\mathrm{R}-\mathrm{h}^{(1)}-\mathrm{h}^{(2)}-3 \mathrm{~d}-\mathrm{h}^{(3)}}=\sigma_{\mathrm{zz}}^{(5), 0} \frac{\mathrm{d} \delta\left(\mathrm{t}_{3}\right)}{\mathrm{dt}_{3}} \cos \theta, \\
& \left.\sigma_{\mathrm{rr}}^{(5), 1}\right|_{\mathrm{r}=\mathrm{R}-\mathrm{h}^{(1)}-\mathrm{h}^{(2)}-\mathrm{h}^{(3)}-\mathrm{h}^{(4)}-3 \mathrm{~d}}=0, \\
& \left.\sigma_{\mathrm{r} \theta}^{(5), 1}\right|_{\mathrm{r}=\mathrm{R}-\mathrm{h}^{(1)}-\mathrm{h}^{(2)}-\mathrm{h}^{(3)}-\mathrm{h}^{(4)}-3 \mathrm{~d}}=0 \\
& \left.\sigma_{\mathrm{rz}}^{(5), 1}\right|_{\mathrm{r}=\mathrm{R}-\mathrm{h}^{(1)}-\mathrm{h}^{(2)}-\mathrm{h}^{(3)}-\mathrm{h}^{(4)}-3 \mathrm{~d}}=\sigma_{\mathrm{zz}}^{(5), 0} \frac{\mathrm{d} \delta\left(\mathrm{t}_{3}\right)}{\mathrm{dt}_{3}} \cos \theta
\end{aligned}
$$

Çözümü için aşağıdaki gösterilim kullanılacaktır (Güz, 1999).

$$
\begin{aligned}
& u_{r}^{(1)}=\frac{1}{r} \frac{\partial}{\partial \theta} \gamma^{(1)}-\frac{\partial^{2}}{\partial r \partial z} \beta^{(1)} u_{\theta}^{(1)}=-\frac{\partial}{\partial r} \gamma^{(1)}-\frac{1}{r} \frac{\partial^{2}}{\partial \theta \partial z} \beta^{(1)} \\
& u_{z}^{(1)}=\left(\lambda^{(1)}+\mu^{(1)}\right)^{-1}\left(\left(\lambda^{(1)}+2 \mu^{(1)}\right) \Delta_{1}+\left(\mu^{(1)}+\sigma_{z z}^{(1), 0}\right) \frac{\partial^{2}}{\partial z^{2}}\right) \beta^{(k)}
\end{aligned}
$$


$\Delta_{1}=\frac{\partial^{2}}{\partial \mathrm{r}^{2}}+\frac{1}{\mathrm{r}} \frac{\partial}{\partial \mathrm{r}}+\frac{1}{\mathrm{r}^{2}} \frac{\partial^{2}}{\partial \theta^{2}}$

(17) ifadelerindeki $\gamma^{(1)}, \beta^{(1)}$ fonksiyonları

$$
\begin{aligned}
& \left(\Delta_{1}^{(1)}+\left(\xi_{1}^{(1)}\right)^{2} \frac{\partial^{2}}{\partial \mathrm{z}^{2}}\right) \gamma^{(1)}=0 \\
& \left(\Delta_{1}^{(1)}+\left(\xi_{2}^{(1)}\right)^{2} \frac{\partial^{2}}{\partial \mathrm{z}^{2}}\right)\left(\Delta_{1}^{(1)}+\left(\xi_{3}^{(1)}\right)^{2} \frac{\partial^{2}}{\partial \mathrm{z}^{2}}\right) \gamma^{(1)}=0
\end{aligned}
$$

(18)' deki eşitliklerini sağlamaktadır. Burada $\xi_{\mathrm{i}}^{(\mathrm{k})}(\mathrm{k}=1,2$; $\mathrm{i}=1,2,3)$ aşağıdaki şekilde ifade edilebilirler.

$$
\begin{aligned}
& \xi_{1}^{(\mathrm{k})}=\sqrt{\frac{\mu^{(\mathrm{k})}+\sigma_{z z}^{(\mathrm{k}), 0}}{\mu^{(\mathrm{k})}}} \xi_{2}^{(\mathrm{k})}=\sqrt{\frac{\mu^{(\mathrm{k})}+\sigma_{z z}^{(\mathrm{k}), 0}}{\mu^{(\mathrm{k})}}} \\
& \xi_{3}^{(\mathrm{k})}=\sqrt{\frac{\lambda^{(\mathrm{k})}+2 \mu^{(\mathrm{k})}+\sigma_{z z}^{(\mathrm{k}), 0}}{\lambda^{(\mathrm{k})}+2 \mu^{(\mathrm{k})}}}
\end{aligned}
$$

İlgili sınır değer probleminin çözümü için bütün denklemlere

$$
\bar{f}(s)=\int_{-\infty}^{\infty} f(z) e^{-i s z} d z
$$

ile verilen üstel Fourier dönüşümü $z=\frac{x_{3}}{L}$,ye göre uygulanır. Fourier transformasyonu uyguladıktan sonra, (18) diferansiyel denklemleri, denge denklemleri ve temas koşulları göz önünde bulundurulurak çözülürse

$$
\begin{aligned}
& \bar{\gamma}^{(1), 1}=\overline{\mathrm{A}}_{1}^{(1)}(\mathrm{s}) \mathrm{K}_{1}\left(\xi_{1}^{(1)} \mathrm{s} \frac{\mathrm{r}}{\mathrm{L}}\right) \sin \theta \\
& \bar{\beta}^{(1), 1}=\mathrm{i}\left[\overline{\mathrm{A}}_{2}^{(1)}(\mathrm{s}) \mathrm{K}_{1}\left(\xi_{2}^{(1)} \mathrm{s} \frac{\mathrm{r}}{\mathrm{L}}\right)+\overline{\mathrm{A}}_{3}^{(1)}(\mathrm{s}) \mathrm{K}_{1}\left(\xi_{3}^{(1)} \mathrm{s} \frac{\mathrm{r}}{\mathrm{L}}\right)\right] \cos \theta \\
& \bar{\gamma}^{(2), 1}=\left[\overline{\mathrm{B}}_{11}^{(2)}(\mathrm{s}) \mathrm{I}_{1}\left(\xi_{1}^{(2)} \mathrm{s} \frac{\mathrm{r}}{\mathrm{L}}\right)+\overline{\mathrm{B}}_{12}^{(2)}(\mathrm{s}) \mathrm{K}_{1}\left(\xi_{1}^{(2)} \mathrm{s} \frac{\mathrm{r}}{\mathrm{L}}\right)\right] \sin \theta \\
& \bar{\beta}^{(2), 1}=\mathrm{i}\left[\begin{array}{l}
\overline{\mathrm{B}}_{21}^{(2)}(\mathrm{s}) \mathrm{I}_{1}\left(\xi_{2}^{(2)} \mathrm{s} \frac{\mathrm{r}}{\mathrm{L}}\right)+\overline{\mathrm{B}}_{22}^{(2)}(\mathrm{s}) \mathrm{K}_{1}\left(\xi_{2}^{(2)} \mathrm{s} \frac{\mathrm{r}}{\mathrm{L}}\right) \\
+\overline{\mathrm{B}}_{31}^{(2)}(\mathrm{s}) \mathrm{I}_{1}\left(\xi_{3}^{(2)} \mathrm{s} \frac{\mathrm{r}}{\mathrm{L}}\right)+\overline{\mathrm{B}}_{32}^{(2)}(\mathrm{s}) \mathrm{K}_{1}\left(\xi_{3}^{(2)} \mathrm{s} \frac{\mathrm{r}}{\mathrm{L}}\right)
\end{array}\right] \cos \theta \\
& \bar{\gamma}^{-(3), 1}=\left[\overline{\mathrm{B}}_{11}^{(3)}(\mathrm{s}) \mathrm{I}_{1}\left(\xi_{1}^{(3)} \mathrm{s} \frac{\mathrm{r}}{\mathrm{L}}\right)+\overline{\mathrm{B}}_{12}^{(3)}(\mathrm{s}) \mathrm{K}_{1}\left(\xi_{1}^{(3)} \mathrm{s} \frac{\mathrm{r}}{\mathrm{L}}\right)\right] \sin \theta \\
& \bar{\beta}^{(3), 1}=\mathrm{i}\left[\begin{array}{l}
\overline{\mathrm{B}}_{21}^{(3)}(\mathrm{s}) \mathrm{I}_{1}\left(\xi_{2}^{(3)} \mathrm{s} \frac{\mathrm{r}}{\mathrm{L}}\right)+\overline{\mathrm{B}}_{22}^{(3)}(\mathrm{s}) \mathrm{K}_{1}\left(\xi_{2}^{(3)} \mathrm{s} \frac{\mathrm{r}}{\mathrm{L}}\right) \\
+\overline{\mathrm{B}}_{31}^{(3)}(\mathrm{s}) \mathrm{I}_{1}\left(\xi_{3}^{(3)} \mathrm{s} \frac{\mathrm{r}}{\mathrm{L}}\right)+\overline{\mathrm{B}}_{32}^{(3)}(\mathrm{s}) \mathrm{K}_{1}\left(\xi_{3}^{(3)} \mathrm{s} \frac{\mathrm{r}}{\mathrm{L}}\right)
\end{array}\right] \cos \theta \\
& \bar{\gamma}^{-(4), 1}=\left[\overline{\mathrm{B}}_{11}^{(4)}(\mathrm{s}) \mathrm{I}_{1}\left(\xi_{1}^{(3)} \mathrm{s} \frac{\mathrm{r}}{\mathrm{L}}\right)+\overline{\mathrm{B}}_{12}^{(4)}(\mathrm{s}) \mathrm{K}_{1}\left(\xi_{1}^{(3)} \mathrm{s} \frac{\mathrm{r}}{\mathrm{L}}\right)\right] \sin \theta
\end{aligned}
$$

$$
\begin{aligned}
& \bar{\beta}^{(4), 1}=\mathrm{i}\left[\begin{array}{l}
\overline{\mathrm{B}}_{21}^{(4)}(\mathrm{s}) \mathrm{I}_{1}\left(\xi_{2}^{(3)} \mathrm{s} \frac{\mathrm{r}}{\mathrm{L}}\right)+\overline{\mathrm{B}}_{22}^{(4)}(\mathrm{s}) \mathrm{K}_{1}\left(\xi_{2}^{(3)} \mathrm{s} \frac{\mathrm{r}}{\mathrm{L}}\right) \\
+\overline{\mathrm{B}}_{31}^{(4)}(\mathrm{s}) \mathrm{I}_{1}\left(\xi_{3}^{(3)} \mathrm{s} \frac{\mathrm{r}}{\mathrm{L}}\right)+\overline{\mathrm{B}}_{32}^{(4)}(\mathrm{s}) \mathrm{K}_{1}\left(\xi_{3}^{(3)} \mathrm{s} \frac{\mathrm{r}}{\mathrm{L}}\right)
\end{array}\right] \cos \theta \\
& \bar{\gamma}^{-(4), 1}=\left[\overline{\mathrm{B}}_{11}^{(4)}(\mathrm{s}) \mathrm{I}_{1}\left(\xi_{1}^{(3)} \mathrm{s} \frac{\mathrm{r}}{\mathrm{L}}\right)+\overline{\mathrm{B}}_{12}^{(4)}(\mathrm{s}) \mathrm{K}_{1}\left(\xi_{1}^{(3)} \mathrm{s} \frac{\mathrm{r}}{\mathrm{L}}\right)\right] \sin \theta \\
& \bar{\beta}^{(4), 1}=\mathrm{i}\left[\begin{array}{c}
\overline{\mathrm{B}}_{21}^{(4)}(\mathrm{s}) \mathrm{I}_{1}\left(\xi_{2}^{(3)} \mathrm{s} \frac{\mathrm{r}}{\mathrm{L}}\right)+\overline{\mathrm{B}}_{22}^{(4)}(\mathrm{s}) \mathrm{K}_{1}\left(\xi_{2}^{(3)} \mathrm{s} \frac{\mathrm{r}}{\mathrm{L}}\right) \\
+\overline{\mathrm{B}}_{31}^{(4)}(\mathrm{s}) \mathrm{I}_{1}\left(\xi_{3}^{(3)} \mathrm{s} \frac{\mathrm{r}}{\mathrm{L}}\right)+\overline{\mathrm{B}}_{32}^{(4)}(\mathrm{s}) \mathrm{K}_{1}\left(\xi_{3}^{(3)} \mathrm{s} \frac{\mathrm{r}}{\mathrm{L}}\right)
\end{array}\right] \cos \theta \\
& \bar{\gamma}^{-(5), 1}=\left[\overline{\mathrm{B}}_{11}^{(5)}(\mathrm{s}) \mathrm{I}_{1}\left(\xi_{1}^{(3)} \mathrm{s} \frac{\mathrm{r}}{\mathrm{L}}\right)+\overline{\mathrm{B}}_{12}^{(5)}(\mathrm{s}) \mathrm{K}_{1}\left(\xi_{1}^{(3)} \mathrm{s} \frac{\mathrm{r}}{\mathrm{L}}\right)\right] \sin \theta \\
& \bar{\beta}^{(5), 1}=\mathrm{i}\left[\begin{array}{l}
\overline{\mathrm{B}}_{21}^{(5)}(\mathrm{s}) \mathrm{I}_{1}\left(\xi_{2}^{(3)} \mathrm{s} \frac{\mathrm{r}}{\mathrm{L}}\right)+\overline{\mathrm{B}}_{22}^{(5)}(\mathrm{s}) \mathrm{K}_{1}\left(\xi_{2}^{(3)} \mathrm{s} \frac{\mathrm{r}}{\mathrm{L}}\right) \\
+\overline{\mathrm{B}}_{31}^{(5)}(\mathrm{s}) \mathrm{I}_{1}\left(\xi_{3}^{(3)} \mathrm{s} \frac{\mathrm{r}}{\mathrm{L}}\right)+\overline{\mathrm{B}}_{32}^{(5)}(\mathrm{s}) \mathrm{K}_{1}\left(\xi_{3}^{(3)} \mathrm{s} \frac{\mathrm{r}}{\mathrm{L}}\right)
\end{array}\right] \cos \theta
\end{aligned}
$$

elde edilir. Burada $K_{n}(x)$ Macdonald ve $I_{n}(x)$ Bessel fonksiyonu fonksiyonudur. (21) fonksiyonları ilgili yerlerde yerine yazıldı̆̆ında $27 \times 27$ lineer denklem sistemi elde edilir. $\mathrm{Bu}$ sistem çözüldügünde $\overline{\mathrm{A}}_{1}^{(1)}(\mathrm{s}), \overline{\mathrm{A}}_{2}^{(1)}(\mathrm{s}), \overline{\mathrm{B}}_{21}^{(4)}(\mathrm{s}) \ldots ., \overline{\mathrm{B}}_{32}^{(5)}(\mathrm{s})$ bilinmeyenleri elde edilerek, gerilme değerlerinin fourier dönüşümlü hallerine ulaşılmış olur. Daha sonra ters fourier dönüşümü uygulanarak gerçek değerler elde edilir.

\section{Araştırma Sonuçları ve Tartışma}

Gerilme analiziyle ile ilgili çalışmalar $\sigma_{\mathrm{nt}}, \sigma_{\mathrm{ne}}, \sigma_{\mathrm{te}}$ kayma gerilmeleri ve $\sigma_{\mathrm{nn}}, \sigma_{\mathrm{ee}}, \sigma_{\tau \tau}$ normal gerilmelerine çeşitli parametrelere bağlı nümerik sonuçlar elde etmek ve bu sonuçların yorumlanması kapsamında yapılmıştır. Bu gerilmeler, matris ve karbon nanotüp arakesit yüzeyi $\mathrm{S}_{8}$ üzerinde $\mathbf{n}$ birim normal vektörü ve $\tau$, $\mathbf{e}$ teğet vektörleri doğrultusundaki ve bu vektörlerin oluşturduğu düzlemlerdeki gerilmelerdir.

Sayısal sonuçlar için $R$ en dış yarıçap, $h^{(1)}$ en dıştaki tüpün kalınlığı, $\mathrm{h}^{(2)}$ dıştan ikinci tüpün kalınlığg, $\mathrm{h}^{(3)}$ dıştan üçüncü tüpün kalınlığı, $\mathrm{h}^{(4)}$ en içteki tüpün kalınlığ 1 ve $\mathrm{d}$ tüpler arasındaki uzaklık olmak üzere $\mathrm{R} / \mathrm{L}, \mathrm{h}^{(1)} / \mathrm{R}, \mathrm{d} / \mathrm{h}^{(1)}$ boyutsuz parametreleri tanımlanmıştır. Karbon nanotüpün duvarlarının kalınlıkları eşit $\mathrm{h}^{(1)}=\mathrm{h}^{(2)}=\mathrm{h}^{(3)}=\mathrm{h}^{(4)}$ kabul edilmiştir. Malzemenin karbon nanotüp olması dikkate alınarak hesaplamalarda kullanılacak olan bu parametreler için aşağıdaki aralıklar belirlenmiştir (Akbarov, 2013: 2587)

$$
\begin{aligned}
& 400 \leq \mathrm{E}^{(2)} / \mathrm{E}^{(1)} \leq 1000,0.015 \leq \mathrm{h}^{(1)} / \mathrm{R} \leq 0.4, \\
& 1 \leq \mathrm{d} / \mathrm{h}^{(1)} \leq 2
\end{aligned}
$$

Tüpler arasında van der Waals kuvvetinin oluşabilmesi için $\mathrm{d} \leq 0.34 \mathrm{~nm}$ olmasi gerekmektedir (Xiaohu ve diğerleri, 2006). $\mathrm{Bu}$ kuvvetleri temsil eden $\mathrm{c}$ sabiti için $0<\mathrm{c} \leq 9.92 \mathrm{TPa}$ aralığ alınmıştır (Akbarov, 2013: 2587). Bundan başka $F$ parametresi ile van der Waals kuvvetlerinin parametreler üzerindeki etkisi ölçülmüştür ve $\mathrm{F}$ aşağıdaki gibi tanımlanmıştır: 


$$
\mathrm{F}=\frac{\mu_{\mathrm{CNT}}}{\mathrm{c}}\left(1-\frac{\mathrm{h}^{(1)}}{\mathrm{R}}\right)
$$

$\mathrm{F}=0$ olduğunda karbon nanotüpün tüpleri arasındaki van der Waals kuvveti sıfırdır ve tüplerin radyal doğrultuda yaptığı yer değiştirme birbirine eşittir. $F=\infty$ ise karbon nanotüpün tüpleri arasinda temas $(\mathrm{d}>0.34 \mathrm{~nm})$ yok demektir (Akbarov, 2013). Burada $\mu_{\mathrm{CNT}}$ parametresinde geçen CNT simgesi karbon nanotüpü temsil etmektedir ve $\left.\mu_{\mathrm{CNT}}=\mu^{(2)}=\mu^{(3)}\right)$. Ayrıca hesaplamalarda $v^{(1)}=v^{(2)}=v^{(3)}=v^{(4)}=v^{(5)}=0.3, \quad \varepsilon=0.07 \quad$ olarak kullanılmıştır.

Gerilmelerin R/L parametresine göre değişimini gösteren tablolarda ve grafiklerde $\sigma_{\mathrm{nn}}, \sigma_{\mathrm{ne}}, \sigma_{\tau \tau}, \sigma_{\mathrm{ee}}$ için $\theta=0 ; \sigma_{\mathrm{n \tau}}$ ve $\sigma_{\tau \text { e }}$ için $\theta=\pi / 2$ değeri kullanılmıştır. Şekil 2 grafiklerinde $\mathrm{h} / \mathrm{R}=0.125, \mathrm{~d} / \mathrm{h}=1, \mathrm{E}^{(2)} / \mathrm{E}^{(1)}=500$ ve $\mathrm{F}=0$ değerlerinde $\sigma_{\mathrm{nn}} /|\mathrm{p}|, \quad \sigma_{\mathrm{ne}} /|\mathrm{p}| \quad$ ile $\mathrm{R} / \mathrm{L}$ parametresi arasındaki ilişki görülmektedir. Bu grafiklerden, $\sigma_{\mathrm{nn}} /|\mathrm{p}|, \sigma_{\mathrm{ne}} /|\mathrm{p}|$ gerilmelerinin mutlak değerinin nanotüpün dış yarıçapı ile olan ilişkisinin monoton olmadığı izlenmektedir. Bu sonuç, iki ve üç duvarlı yerel eğrilikli CNT sonuçlarıyla benzerdir (Coban, 2016).

Tablo 1'de sirasi ile $\sigma_{\mathrm{nn}} /|\mathrm{p}|, \quad \sigma_{\mathrm{n \tau}} /|\mathrm{p}|, \quad \sigma_{\mathrm{ne}} /|\mathrm{p}|, \quad \sigma_{\tau \tau} /|\mathrm{p}|$, $\sigma_{\text {тe }} /|\mathrm{p}|, \quad \sigma_{\text {ee }} /|\mathrm{p}| \quad$ değerleri çeşitli $\mathrm{m} \quad$ ve $\mathrm{E}^{(1)} / \mathrm{E}^{(2)}$ parametrelerine göre verilmiştir. $\mathrm{Bu}$ çizelgelerden $\mathrm{E}^{(2)} / \mathrm{E}^{(1)}$ değeri büyüdükçe $\sigma_{\mathrm{nn}} /|\mathrm{p}|, \quad \sigma_{\mathrm{ne}} /|\mathrm{p}|, \sigma_{\mathrm{n \tau}} /|\mathrm{p}|, \sigma_{\mathrm{ee}} /|\mathrm{p}|, \sigma_{\tau \tau} /|\mathrm{p}|$ gerilmelerinin de mutlak değerce büyüdüğü izlenmektedir. $\sigma_{\tau e} /|\mathrm{p}|$ gerilmesinin değerleri ise aynı durumda düşmektedir. Not edilmelidir ki $\sigma_{\mathrm{n \tau}} /|\mathrm{p}|$ değerlerinde artış diğer gerilmelere göre daha azdır ve $\sigma_{\mathrm{ee}} /|\mathrm{p}|$ gerilmesinin değeri $\mathrm{m}=3$ 'de daha fazla düşüş göstermektedir. $\mathrm{E}^{(2)} / \mathrm{E}^{(1)}$ ve gerilme değerleri arasındaki ilişki literatür ile uyumludur. İki ve üç duvarlı yerel eğrilikli CNT için de sonuçlar benzerdir (Coban, 2016).

Tablo 2'de sirasi ile $\sigma_{\text {nn }} /|\mathrm{p}|, \quad \sigma_{\text {ne }} /|\mathrm{p}|, \quad \sigma_{\mathrm{n \tau}} /|\mathrm{p}|, \sigma_{\mathrm{ee}} /|\mathrm{p}|$, $\sigma_{\tau \tau} /|\mathrm{p}|$ değerleri çeşitli $\mathrm{m}$ ve $\mathrm{F}$ parametrelerine göre verilmiştir. Bu çizelgelerden F değeri büyüdükçe $\sigma_{\mathrm{nn}} /|\mathrm{p}|, \sigma_{\mathrm{ne}} /|\mathrm{p}|, \sigma_{\mathrm{n \tau}} /|\mathrm{p}|$, $\sigma_{\mathrm{ee}} /|\mathrm{p}|, \quad \sigma_{\tau \tau} /|\mathrm{p}| \quad$ gerilmelerinin mutlak değerce azaldı̆̆ görülmektedir. $\sigma_{\tau e} /|\mathrm{p}|$ gerilmesinin değerleri ise aynı durumda artmaktadır. Ayrıca $\sigma_{\text {ee }} /|\mathrm{p}|$ gerilmesinin değeri $\mathrm{m}=3$ 'de daha fazla düşüş göstermektedir.
$\mathrm{Bu}$ grafikler ve tablolarda sıfirıncı ve birinci yaklaşım sonucunda elde edilen gerilme değerlerinin sayısal sonuçları görülmektedir. F değerinin arttıkça gerilme değerlerinin düşmesi literatür ile uyumlu bir sonuçtur. İki ve üç duvarlı yerel eğrilikli CNT için de sonuçlar benzerdir (Coban, 2016).
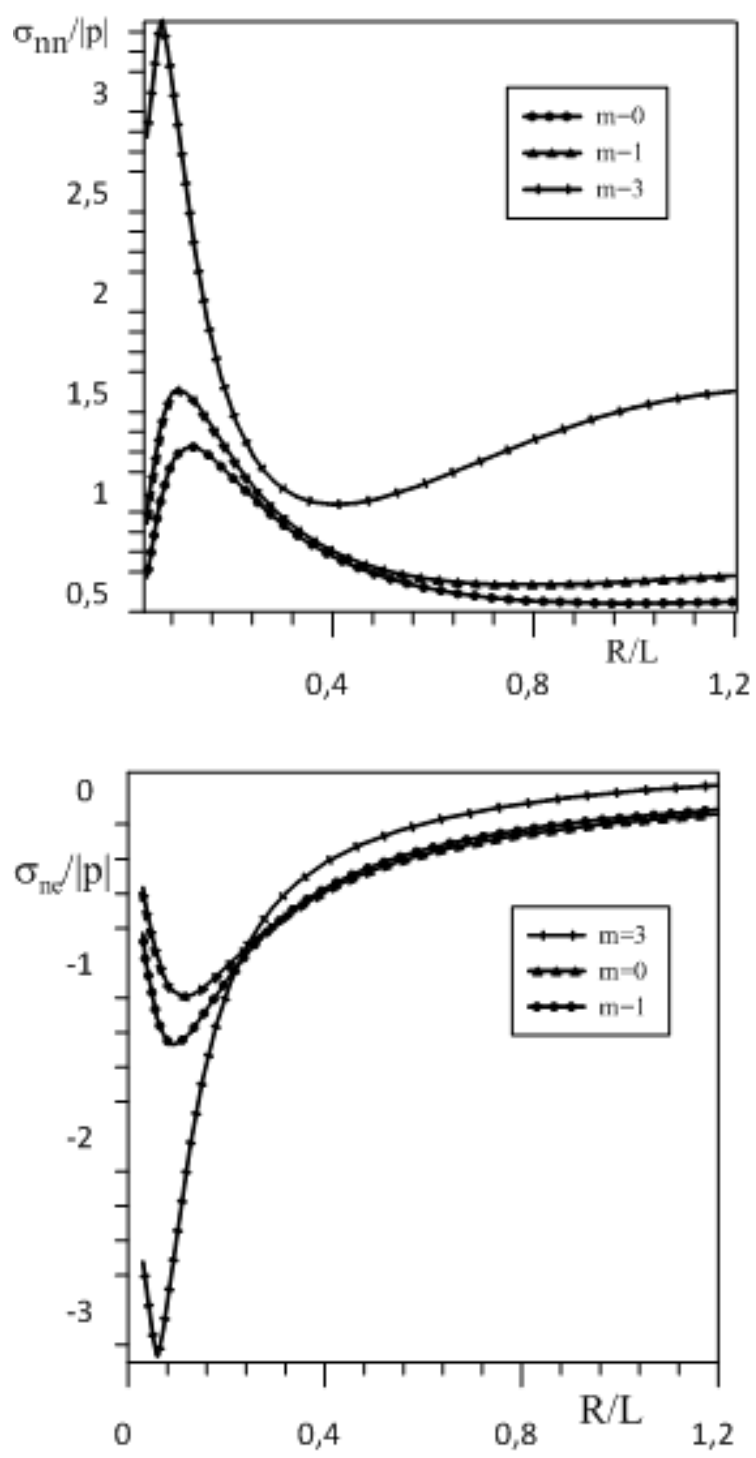

Şekil 2. Çeşitli m değerleri için (a) $\sigma_{\mathrm{nn}} /|\mathrm{p}|$ (b) $\sigma_{\mathrm{ne}} /|\mathrm{p}|$ ve R/L arasındaki bağımlılık

Tablo 1. Çeşitli $\mathrm{E}^{(2)} / \mathrm{E}^{(1)}$ ve m değerleri için, $\sigma_{\mathrm{nn}} /|\mathrm{p}|, \sigma_{\mathrm{n \tau}} /|\mathrm{p}|, \sigma_{\mathrm{ne}} /|\mathrm{p}|, \sigma_{\tau \tau} /|\mathrm{p}|, \sigma_{\mathrm{re}} /|\mathrm{p}|, \sigma_{\mathrm{ee}} /|\mathrm{p}|$ değgerleri $(F=100, R / L=0.6, \mathrm{~h} / \mathrm{R}=0.125$, $\mathrm{d} / \mathrm{h}=1)$

\begin{tabular}{|c|c|c|c|c|c|c|c|}
\hline \multirow{2}{*}{$\mathrm{E}^{(2)} / \mathrm{E}^{(1)}$} & $\mathrm{m}$ & $\sigma_{\mathrm{nn}} /|\mathrm{p}|$ & $\sigma_{\mathrm{n \tau}} /|\mathrm{p}|$ & $\sigma_{\mathrm{ne}} /|\mathrm{p}|$ & $\sigma_{\tau \tau} /|\mathrm{p}|$ & $\sigma_{\tau \mathrm{e}} /|\mathrm{p}|$ & $\sigma_{\mathrm{ee}} /|\mathrm{p}|$ \\
\hline \multirow{3}{*}{500} & 0 & 0.51325 & 0.23430 & -0.37296 & 0.99780 & 0.01653 & 0.12954 \\
\cline { 2 - 8 } & 1 & 0.56183 & 0.26640 & $-0,34719$ & 0.98609 & 0.02017 & 0.11174 \\
\cline { 2 - 8 } & 3 & 1,05739 & 0.22483 & $-0,19617$ & 0.95862 & 0.01548 & 0.01520 \\
\hline 700 & 0 & 0.56581 & 0.24375 & $-0,42284$ & 1,00703 & 0.01432 & 0.14940 \\
\hline
\end{tabular}


Avrupa Bilim ve Teknoloji Dergisi

\begin{tabular}{|c|c|c|c|c|c|c|c|}
\hline \multirow{3}{*}{1000} & 1 & 0.60654 & 0.27501 & $-0,38853$ & 0.99333 & 0.01794 & 0.12811 \\
\cline { 2 - 8 } & 3 & 1,07730 & 0.22831 & $-0,20639$ & 0.95976 & 0.01392 & 0.01874 \\
\hline \multirow{3}{*}{10} & 0 & 0.62354 & 0.25275 & -0.47831 & 1,01859 & 0.01241 & 0.17150 \\
\cline { 2 - 8 } & 1 & 0.65471 & 0.28302 & -0.43393 & 1,00244 & 0.01605 & 0.14613 \\
\cline { 2 - 8 } & 3 & 1,09480 & 0.23124 & -0.21638 & 0,96118 & 0.01268 & 0.02234 \\
\hline
\end{tabular}

Tablo 2. Çeşitli F ve m değerleri için, $\sigma_{\mathrm{nn}} /|\mathrm{p}|, \sigma_{\mathrm{n \tau}} /|\mathrm{p}|, \sigma_{\mathrm{ne}} /|\mathrm{p}|, \sigma_{\tau \tau} /|\mathrm{p}|, \sigma_{\tau \mathrm{re}} /|\mathrm{p}|, \sigma_{\mathrm{ee}} /|\mathrm{p}|$ değerleri $\left(\mathrm{E}^{(2)} / \mathrm{E}^{(1)}=700, R / L=0.6\right.$, $\mathrm{h} / \mathrm{R}=0.125, \mathrm{~d} / \mathrm{h}=1)$

\begin{tabular}{|c|c|c|c|c|c|c|c|}
\hline $\mathrm{F}$ & $\mathrm{m}$ & $\sigma_{\mathrm{nn}} /|\mathrm{p}|$ & $\sigma_{\mathrm{n} \tau} /|\mathrm{p}|$ & $\sigma_{\mathrm{ne}} /|\mathrm{p}|$ & $\sigma_{\tau \tau} /|\mathrm{p}|$ & $\sigma_{\tau \mathrm{e}} /|\mathrm{p}|$ & $\sigma_{\mathrm{ee}} /|\mathrm{p}|$ \\
\hline \multirow{3}{*}{100} & 0 & 0,56581 & 0,24375 & $-0,42284$ & 1,00703 & 0,01432 & 0,14940 \\
\cline { 2 - 8 } & 1 & 0,60654 & 0,27501 & $-0,38853$ & 0,99333 & 0,01794 & 0,12811 \\
\cline { 2 - 8 } & 3 & 1,07730 & 0,22831 & $-0,20639$ & 0,95976 & 0,01392 & 0,01874 \\
\hline \multirow{3}{*}{300} & 0 & 0,52742 & 0,23523 & $-0,38950$ & 1,00275 & 0,01650 & 0,13853 \\
\cline { 2 - 8 } & 1 & 0,57426 & 0,26766 & $-0,36056$ & 0,98984 & 0,01988 & 0,11900 \\
\cline { 2 - 8 } & 3 & 1,07202 & 0,22628 & $-0,20001$ & 0,95936 & 0,01450 & 0,01665 \\
\hline \multirow{3}{*}{500} & 0 & 0,51129 & 0,23222 & $-0,37496$ & 1,00048 & 0,01716 & 0,13357 \\
\cline { 2 - 8 } & 1 & 0,56097 & 0,26512 & $-0,34859$ & 0,98800 & 0,02046 & 0,11493 \\
\cline { 2 - 8 } & 3 & 1,06975 & 0,22567 & $-0,19761$ & 0,95909 & 0,01466 & 0,01582 \\
\hline
\end{tabular}

\section{Sonuç}

Bu çalışmada günümüz teknolojisinde çok önemli yere sahip olan nanomalzemelerin en önemli üyesi olarak kabul edilen karbon nanotüpün dört katlı olarak elastik matris malzemesi içine gömülmesiyle elde edilen kompozit malzemenin düzgün yayılmış normal kuvvetler altındaki gerilme analizi ile ilgili çeşitli parametrelere bağlı sonuçları verilmiştir.

Karbon nanotüpler sürekli bir malzeme olarak ele alınmış dolayısıyla sürekli ortam mekaniği kanunlarına göre çözüm aranmıştır. Fiziksel ve matematiksel modellemesi yapılan problemde, karbon nanotüplerin katmanlarının içi boş ortak merkezli dört silindirin içe içe geçmesiyle oluştuğu varsayılmıştır. Katmanlar arasında van der Waals kuvvetlerinin ortaya çıktığı düşünülmüştür. Bahsedilen nanotüpün başlangıç ilkel bir kusuru olduğu kabul edilerek yerel eğrilikli olarak modellenmiş ve hesaplamalar bu kapsamda yapılmıştır. Çalışma kapsamında, ilgili sınır-değer problemleri için elastisite teorisine ait kesin geometrik lineer denklemler kullanılıp, parçalı-homojen cisim modeli sınırları dahilinde matematiksel formülasyonu yapılmıştır. Dört duvarlı karbon nanotüpün üç boyutlu olarak modellenerek yaklaşık analitik bir metotla gerilme dağılımının incelenmesi ilk defa yapılmaktadır. Ayrıca bu araştırmada karbon nanotüplerin arasındaki boşluğu ifade eden d uzaklı̆ğ limit olarak sıfira götürüldüğ̈̈nde, problem tek duvarlı karbon nanotüp problemine dönüşür ve (Coban, 2009)'de elde edilen gerilme değerleri ile aynı sonuca ulaşılmıştır. $\mathrm{Bu}$ da yapılan algoritma ve kodlamanın doğruluğunu gösterir. Ayrıca dört duvarlı CNT için elde edilen gerilme değerleri (Coban, 2016)'de üç katlı için elde edilen değerlerden daha düşüktür. Yine (Coban, 2016)'de üç katlı CNT için elde edilen gerilme değerlerinin iki katlı CNT için elde edilen değerlerden daha düşük olduğu gösterilmiştir. Dolayısıyla CNT' ler için duvar sayısı arttıkça gerilme değerlerinin düşeceği söylenebilir.
Bu çalışmada $\left(\mathrm{E}^{(2)} / \mathrm{E}^{(1)}\right)$ değeri 300,500 ve 1000 olarak alınmıştır ve normal gerilmeler ile kayma gerilmeleri geometrik lineer durumda incelenmiştir. Ulaşılan değerler sıfirıncı ve bir yaklaşımın çözümünden elde edilmiştir. Çalışma ile ilgili sonuçlar şu şekilde özetlenebilir:

- Nanotüpteki duvar sayısı artırıldığında gerilme değerlerinin düştüğü görülmüştür.

- Dört duvarlı karbon nanotüpün dış yarıçapa göre değişimine bakıldı̆̆ında, $\sigma_{\mathrm{nn}} /|\mathrm{p}|$ ve $\sigma_{\text {ne }} /|p|$ gerilmelerinin mutlak değerinin, dış yarıçap ile monoton olmayan şekilde değiştiği görülmektedir.

- Elastisite modülü $\left(\mathrm{E}^{(2)} / \mathrm{E}^{(1)}\right)$ değeri büyüdükçe $\sigma_{\mathrm{nn}} /|\mathrm{p}|, \quad \sigma_{\mathrm{ne}} /|\mathrm{p}|, \quad \sigma_{\mathrm{n \tau}} /|\mathrm{p}|, \quad \sigma_{\mathrm{ee}} /|\mathrm{p}|, \quad \sigma_{\tau \tau} /|\mathrm{p}|$ gerilmelerinin de mutlak değerce büyüdüğü izlenmektedir. $\sigma_{\tau e} /|\mathrm{p}|$ gerilmesinin değerleri ise aynı durumda düşmektedir.

- Van der Waals kuvvetlerinin etkisinin artmasiyla (F ile temsil edilir) $\sigma_{\mathrm{nn}} /|\mathrm{p}|, \sigma_{\mathrm{ne}} /|\mathrm{p}|$ $, \quad \sigma_{\text {n }} /|\mathrm{p}|, \quad \sigma_{\mathrm{ee}} /|\mathrm{p}|, \quad \sigma_{\tau \tau} /|\mathrm{p}|$ gerilmelerinin mutlak değerce azaldığı görülmektedir. $\sigma_{\tau e} /|\mathrm{p}|$ gerilmesinin değerleri ise aynı durumda artmaktadır.

- Karbon nanotüpün orta çizgisinin denkleminde yer alan ve salınım frekansını gösteren $\mathrm{m}$ değerine göre gerilmeler araştırılmıştır. Salınım frekansı ile gerilme değerleri arasında monoton olmayan bir ilişki olduğu görülmüştür. 


\section{Kaynakça}

Akbarov, S.D. (2013). Microbuckling of a doublewalled carbon nanotube embedded in an elastic matrix. International journal of Solids and Structures, 50, 2584-2596

Akbarov, S.D. ve Guz, A.N. (1985). Method of Solving Problems in the Mechanics of Fiber Composites with Curved Structures. Soviet Applied Mechanics, 20(9), 777790. Akbarov, S.D. ve Guz, A.N. (2000). Mechanics of Curved Composites, Amsterdam: Kluwer Academic Publishers.

Akbarov, S.D. ve Kosker, R. (2001). Fiber Bucling in a Viscoelastic Matrix. Mechanics of Composite Materials, 37(4), 299-306.

Akbarov, S.D. ve Kosker, R. (2003a). On a Stresss Analysis in the Infinite Elastic Body with Two Neighbouring Curved Fibers. Composites Part B, 34, 143-150.

Akbarov, S.D. ve Kosker, R. (2003b). Influence of the Interaction Two Neighbouring Curved Fibers. Mechanics of Composite Materials, 39, 165-176.

Akbarov, S.D. ve Kosker, R. (2003c). Stress Distribution Caused by Anti-Phase Periodical Curving of Two Neighbouring Fibers in a Composite Materials. European Journal of Mechanics A/Solids, 22, 243-256.

Akbarov, S.D. ve Kosker, R. (2004). Internal Stability Loss of Two Neighboring Fibers in a iscoelastic Matrix. Internal Journal of Engineering Science, 42, 1847-1873.

Akbarov, S.D., Kosker, R. and Cinar, N. (2011). Stress Distribution in an Infinite Body Containing Two Neighboring Locally Curved Nanofibers, CMC-Computers, Materials\&Continua, 21(2), 119-146.

Akbarov, S.D., Kosker, R. and Ucan, Y. (2006). Stress Distribution in a Composite Material With the Row of AntiPhase Periodically Curved Fibres, International Applied Mechanics, 42 (4), 486-493.

Arefi, A. ve Nahvi, H. (2017). Stability analysis of an embedded single-walled carbon nanotube with small initial curvature based on nonlocal theory. Mechanics of Advanced Materials and Structures, 24(11), 962-970.

Breuer, O. ve Sundararaj, U. (2004). Big Returns From Small Fibers: A Review of Polymer/Carbon Nanotube Composites. Polymer Composites, 25(6), 630-645.

Coban, F. (2009). İçi Boş Yerel Ĕgrilikli Tek Lif İçeren Sonsuz Elastik Ortamda Gerilme Dağılımı. (Yayımlanmamış Yüsek Lisans Tezi). Yıldız Teknik Universitesi, İstanbul.

Coban, F. (2016) Yerel Eğrilikli İki ve Üç Duvarlı Karbon Nanotüplerin Gerilme Ve Stabilite Analizi. (Yayımlanmamış Doktora Tezi). Yıldız Teknik Universitesi, İstanbul.

Duan, H.L., Wang, J., ve Karihaloo, B.L. (2009). Theory of elasticity at the nanoscale. Advanced Applied Mechanics, 42(1), 1-68.
Guz, A.N. (1999). Fundamentals of the Three-Dimensional Theory of Stability of Deformable Bodies, Berlin: SpringerVerlag.

Guz, I.A. (2012). Continuum solid mechanics at nano-scale: how small can it go? Journal of Nanomaterials and Molecular Nanotechnology, 1 (1).

Iijima, S. (1991). Helical microtubules of graphitic carbon. Nature, 354, 56-58. International Journal of Solids and structures, 43:, 6832-6854

Kalamkarov, A.L., Georgiades, A.V., Rokam, S.K., Veedu, V.P. ve Ghasemi-Nejhad, M.N. (2006). Analytical and numerical techniques to predict carbon nanotubes properties.

Karličić, D., Kozić, P., Pavlović, R. ve Nešić, N. (2017). Dynamic stability of single-walled carbon nanotube embedded in a viscoelastic medium under the influence of the axially harmonic load. Composite Structures, 162, 227243.

Mantazeri, A. ve Naghdabadi, R. (2009). Study the Effect of Viscoelastic Matrix Model on the Stability of CNT/Polymer Composites by Multiscale Modeling. Polymer Composites, 30(11), 1545-1551.

$\mathrm{Ru}$, C.Q. (2001). Axially compressed buckling of a double walled carbon nanotube embedded in an elastic medium. Journal of Mechanics, Physics and Solids, 49, 1265-1279

Shi, J.X., Natsuki, T., Lei, X.W. ve Ni, Q.Q. (2012). Buckling Instability of Carbon Nanotube Atomic Force Microscope Probe Clamped in an Elastic Medium, Journal of Nanotechnology in Engineering and Medicine. 3(2), 1-5.

Xiaohu, Y. ve Qiang, H. (2006). Investigation of Axially Compressed Buckling of a Multi-Walled Carbon Nanotube Under Temprature Field. Composite Science and Technology, 67, 125-134.

Yan Y, Wang W.Q. ve Zhang, L.X. (2010) Nonlocal effect on axially compressed buckling of triple-walled carbon nanotubes under temperature field. Applied Mathematical Modelling, 34, 3422-3429.

Zhang, M. ve Li, J. (2009). Carbon Nanotube in Different Shapes. Materials Today, 12(6), 12-18. 